\title{
Better Algorithm of Ordinal Online Schedule for Jobs with Similar Sizes on Two Machines
}

\author{
Limin Wang1, Rongheng $\mathrm{Li}^{1 *}$, Yunxia Zhou ${ }^{2}$ \\ ${ }^{1}$ Key Laboratory of Computing and Stochastic Mathematics (Ministry of Education), Department of Mathematics, \\ School of Mathematics and Statistics, Hunan Normal University, Changsha, China \\ ${ }^{2}$ College of Information Science and Engineering, Hunan Normal University, Changsha, China \\ Email: *lirongheng@hunnu.edu.cn
}

How to cite this paper: Wang, L.M., Li, R.H. and Zhou, Y.X. (2019) Better Algorithm of Ordinal Online Schedule for Jobs with Similar Sizes on Two Machines. American Journal of Operations Research, 9, 235-243.

https://doi.org/10.4236/ajor.2019.95015

Received: June 5, 2019

Accepted: September 17, 2019

Published: September 20, 2019

Copyright $\odot 2019$ by author(s) and Scientific Research Publishing Inc. This work is licensed under the Creative Commons Attribution International License (CC BY 4.0).

http://creativecommons.org/licenses/by/4.0/

\begin{abstract}
Ordinal online schedule for jobs with similar sizes in $[1, r](1 \leq r \leq 2)$ on two parallel machines system is considered. Firstly it is proved that the worst case performance ratio of the existing algorithm $P_{2}$ cannot be improved even if the job processing times are known in $[1, r]$ for any $r \geq 1$. Then a better algorithm named $S$ is developed and its worst case performance ratio is given for $1 \leq r \leq 2$.
\end{abstract}

\section{Keywords}

Semi-Online Scheduling, $P_{m}$ Algorithm, S Algorithm, Worst Performance Ratio

\section{Introduction}

The scheduling problem on $m$ parallel identical machines is defined as follows: Given a job set $L=\left\{J_{1}, J_{2}, \cdots, J_{n}\right\}$ of $n$ jobs where job $J_{j}$ has non-negative processing time $p_{j}$, assign the jobs on $m$ machines $\left\{M_{1}, M_{2}, \cdots, M_{m}\right\}$ so as to minimize the maximum completion times of the jobs on each machine. The earliest algorithm for on-line scheduling jobs on parallel machines is the List Scheduling (LS) algorithm, which was introduced by Graham [1]. Many models and algorithms for online scheduling are proposed later on. In classic scheduling problem, there is no constraints on the size of job. However, in practice, the size of job can neither be too large nor too small. This motivates researchers to study scheduling problems when the sizes of all jobs are known in $[1, r]$ with $r \geq 1$ [2]-[7].

In this paper, we will consider ordinal online scheduling jobs with sizes in 
$[1, r](1 \leq r \leq 2)$ on two parallel machines. The model of ordinal online scheduling was proposed by Liu et al. [8]. It is assumed that the values of the processing times are unknown, but that the order of the jobs by non-increasing processing time is known, i.e., without loss of generality that $p_{1} \geq p_{2} \geq \cdots \geq p_{n}$. An algorithm named $P_{m}$ was developed for the system of $m$ machines and it is proved that the algorithm is the best online algorithm for $m=2,3$. In current research, it will be proved that, for $m=2$, the worst case performance ratio of algorithm $P_{2}$ can not be improved even if the sizes of all jobs are known in $[1, r]$ for any $r \geq 1$. Then a better algorithm named $S$ is proposed for $r \in[1,2]$ and its worst case performance ratio is given.

The rest of the paper is organized as follows. In Section 2, some definitions and the algorithm $S$ and $P_{2}$ are given. Section 3 analyzes the competitive ratio of the algorithm S. Finally, some concluding remarks are given in Section 4.

\section{Some Definitions and Algorithms}

Definition 1. Given $m$ parallel machines, let $L=\left\{J_{1}, J_{2}, \cdots, J_{n}\right\}$ be any list of jobs. Algorithm $A$ is a heuristic algorithm. Let $C_{\max }^{A}(L)$ and $C_{\max }^{O P T}(L)$ be the makespan of algorithm $A$ and the makespan of an optimal off-line algorithm respectively. We refer to

$$
R(m, A)=\sup _{L} \frac{C_{\max }^{A}(L)}{C_{\max }^{O P T}(L)}
$$

as the worst case performance ratio of algorithm $A$.

In the following of this paper, we always assume that the number of machines is two (i.e. $m=2$ ) and the sizes of job list $L=\left\{J_{1}, J_{2}, \cdots, J_{n}\right\}$ satisfies $p_{1} \geq p_{2} \geq \cdots \geq p_{n}$ and $p_{j} \in[1, r](j=1,2, \cdots, n, 1 \leq r \leq 2)$ if no specific explanation is given.

Algorithm $P_{2}$ [8]. Jobs are assigned to machines as follows:

$$
\begin{aligned}
& M_{1}:\left\{J_{3 k+1} \mid k \geq 0\right\}, \\
& M_{2}:\left\{J_{3 k-1}, J_{3 k} \mid k \geq 1\right\} .
\end{aligned}
$$

i.e.

$$
\begin{array}{lllllll}
M_{1}: J_{1} & J_{4} & & J_{7} & & J_{10} \cdots \\
M_{2}: J_{2} & J_{3} & J_{5} & J_{6} & J_{8} & J_{9} \cdots
\end{array}
$$

\section{Algorithm $S$.}

Jobs are assigned to machines as follows:

$$
\begin{aligned}
& M_{1}:\left\{J_{1}\right\} \bigcup\left\{J_{4 k}, J_{4 k+1} \mid k \geq 1\right\}, \\
& M_{2}:\left\{J_{4 k-2}, J_{4 k-1} \mid k \geq 1\right\} .
\end{aligned}
$$

i.e.

$$
\begin{array}{cccccc}
M_{1}: J_{1} & J_{4} & J_{5} & J_{8} & J_{9} & J_{12} \cdots \\
M_{2}: J_{2} & J_{3} & J_{6} & J_{7} & J_{10} & J_{11} \cdots
\end{array}
$$

The two algorithms are the same for assigning the first four jobs. The dif- 
ferences are that $P_{2}$ assign the first two jobs on $M_{2}$ and the third on $M_{1}$ for job set $\left\{J_{j} \mid j=3 k+2,3 k+3,3 k+4\right\}(k \geq 1)$. However algorithm $S$ assign the two consecutive job $J_{2 k+1}, J_{2 k+2}(k \geq 2)$ on two machines alteratively.

In the following, we consider the worst case performance ratio of algorithm $P_{2}$ and $S$. We will show that algorithm $S$ is better than $P_{2}$ under the assumption of $p_{j} \in[1, r]$ for $r \leq 2$.

\section{Main Results}

Theorem 1. For algorithm $P_{2}$, its worst case performance ratio is $\frac{4}{3}$. Furthermore, its worst case performance ratio can not be improved if $p_{j}(j=1,2, \cdots, n)$ satisfy $p_{j} \in[1, r]$ for any $r \geq 1$.

Proof: The first conclusion is a direct result from Liu et al. [8]. For the second conclusion, consider job list $L=\left\{J_{1}, J_{2}, \cdots, J_{6 k+4}\right\}$ satisfying

$$
p_{1}=p_{2}=p_{3}=p_{4}=r \geq 1, p_{5}=p_{6}=\cdots=p_{6 k+4}=1 \text {. }
$$

By the rules of $P_{2}$, we get

$$
\begin{array}{lllllll}
M_{1}: r & r & & 1 & & 1 \cdots \\
M_{2}: r & r & 1 & 1 & 1 & 1 \cdots
\end{array}
$$

It is obvious that $C_{\max }^{P_{2}}(L)=2 r+4 k$ and $C_{\max }^{O P T}(L)=2 r+3 k$. Hence

$$
\frac{C_{\max }^{P_{2}}(L)}{C_{\max }^{O P T}(L)}=\frac{2 r+4 k}{2 r+3 k} \rightarrow \frac{4}{3}(k \rightarrow \infty) .
$$

In the following of this paper, let $L_{i}(i=1,2)$ to denote the completion time of machine $M_{i}$ in the schedule assigned by algorithm $S$.

Lemma 2. Given any job list $L=\left\{J_{1}, J_{2}, \cdots, J_{n}\right\}$, the following inequality holds

$$
\frac{C_{\max }^{S}(L)}{C_{\max }^{O P T}(L)} \leq 1+\frac{r}{2 C_{\max }^{O P T}(L)} .
$$

Proof: By the rules of $S$ algorithm, we get

$$
\begin{aligned}
\frac{C_{\max }^{S}(L)}{C_{\max }^{O P T}(L)} & =\frac{\max \left\{L_{1}, L_{2}\right\}}{C_{\max }^{O P T}(L)} \\
& =\frac{L_{1}+L_{2}+\max \left\{L_{1}, L_{2}\right\}-\min \left\{L_{1}, L_{2}\right\}}{2 C_{\max }^{O P T}(L)} \\
& \leq 1+\frac{\left|L_{1}-L_{2}\right|}{2 C_{\max }^{O P T}(L)} .
\end{aligned}
$$

That means it is enough to prove $\left|L_{1}-L_{2}\right| \leq r$. We consider it according to the four cases of $n=4 k, n=4 k+1, n=4 k+2, n=4 k+3$.

Case 1: $n=4 k$. In this case,

$$
L_{1}=p_{1}+\sum_{j=1}^{k-1}\left(p_{4 j}+p_{4 j+1}\right)+p_{4 k} ; L_{2}=\sum_{j=1}^{k}\left(p_{4 j-2}+p_{4 j-1}\right) .
$$

Hence the following inequalities hold: 


$$
\begin{aligned}
& L_{1}-L_{2}=p_{1}+\sum_{j=1}^{k-1}\left(p_{4 j}+p_{4 j+1}-p_{4 j-2}-p_{4 j-1}\right)+p_{4 k}-p_{4 k-2}-p_{4 k-1} \leq p_{1} \leq r, \\
& L_{2}-L_{1}=p_{2}+p_{3}-p_{1}+\sum_{j=1}^{k-1}\left(p_{4 j+2}+p_{4 j+3}-p_{4 j}-p_{4 j+1}\right)-p_{4 k} \leq p_{2} \leq r .
\end{aligned}
$$

That means $\left|L_{1}-L_{2}\right| \leq r$.

Case 2: $n=4 k+1$. In this case,

$$
L_{1}=p_{1}+\sum_{j=1}^{k}\left(p_{4 j}+p_{4 j+1}\right), L_{2}=\sum_{j=1}^{k}\left(p_{4 j-2}+p_{4 j-3}\right) .
$$

Hence the following inequalities hold:

$$
\begin{aligned}
& L_{1}-L_{2}=p_{1}+\sum_{j=1}^{k}\left(p_{4 j}+p_{4 j+1}-p_{4 j-2}-p_{4 j-1}\right) \leq p_{1} \leq r, \\
& L_{2}-L_{1}=p_{2}+p_{3}-p_{1}+\sum_{j=1}^{k-1}\left(p_{4 j+2}+p_{4 j+3}-p_{4 j}-p_{4 j+1}\right)-p_{4 k}-p_{4 k+1} \leq p_{2} \leq r .
\end{aligned}
$$

That means $\left|L_{1}-L_{2}\right| \leq r$. Similarly it is easy to show that the conclusion is true for the case of $n=4 k+2$ and $n=4 k+3$.

Theorem 3. For any job list $L=\left\{J_{1}, J_{2}, \cdots, J_{n}\right\}$ with $p_{1} \geq p_{2} \geq \cdots \geq p_{n}$ and $p_{j} \in[1, r](1 \leq r \leq 2)$, algorithm $S$ has worst case performance ratio

$$
\frac{C_{\max }^{S}(L)}{C_{\max }^{O P T}(L)} \leq \begin{cases}\frac{2+r}{3}, & \frac{3}{2}<r \leq 2 \\ \frac{7}{6}, & \frac{4}{3} \leq r \leq \frac{3}{2} \\ \frac{r+1}{2}, & 1 \leq r<\frac{4}{3}\end{cases}
$$

Proof: Suppose (1) is not true. For $\frac{3}{2} \leq r \leq 2$ the following inequalities hold by Lemma 2 :

$$
\frac{2+r}{3}<\frac{C_{\max }^{S}(L)}{C_{\max }^{O P T}(L)} \leq 1+\frac{r}{2 C_{\max }^{O P T}(L)}
$$

That means $C_{\max }^{O P T}(L)<\frac{3 r}{2(r-1)}<5$. Similarly $C_{\max }^{O P T}(L)<5$ also holds for $\frac{4}{3} \leq r<\frac{3}{2}$. That means there are at most four jobs assigned on any machine in any optimal schedule, i.e., $n \leq 8$. It is easy to prove that algorithm $S$ is optimal if $n<5$. Now consider $n=5$. In this case

$$
\begin{aligned}
& L_{1}=p_{1}+p_{4}+p_{5} \geq p_{2}+2 \geq p_{2}+p_{3}=L_{2}, \\
& C_{\max }^{O P T}(L) \geq p_{3}+p_{4}+p_{5} .
\end{aligned}
$$

Hence

$$
\frac{C_{\max }^{S}(L)}{C_{\max }^{O P T}(L)} \leq \frac{p_{1}+p_{4}+p_{5}}{p_{3}+p_{4}+p_{5}} \leq \frac{p_{1}+\left(p_{4}+p_{5}\right)}{p_{3}+\left(p_{4}+p_{5}\right)} \leq \frac{p_{1}+2}{p_{3}+2} \leq \frac{r+2}{3} .
$$

For the case of $n=6$, there are exactly three jobs on each machine in any optimal schedule. If $L_{1}>L_{2}$, we get 


$$
\frac{C_{\max }^{S}(L)}{C_{\max }^{O P T}(L)} \leq \frac{p_{1}+p_{4}+p_{5}}{p_{1}+p_{5}+p_{6}} \leq \frac{p_{4}+p_{1}+p_{5}}{p_{6}+p_{1}+p_{5}} \leq \frac{r+2}{3}
$$

If $L_{1} \leq L_{2}$ we have

$$
\frac{C_{\max }^{S}(L)}{C_{\max }^{O P T}(L)} \leq \frac{p_{2}+p_{3}+p_{6}}{p_{1}+p_{5}+p_{6}} \leq \frac{p_{2}+\left(p_{3}+p_{6}\right)}{p_{5}+\left(p_{3}+p_{6}\right)} \leq \frac{p_{2}+2}{p_{3}+2} \leq \frac{r+2}{3} .
$$

For the case of $n=7$, the following holds

$$
L_{2}=p_{2}+p_{3}+p_{6}+p_{7} \geq p_{2}+p_{3}+2 \geq p_{1}+p_{2}+p_{3}=L_{1} \text {. }
$$

In any optimal schedule, exactly four jobs are assigned on one machine and exactly three jobs are assigned on another. If the machine assigned four jobs in optimal schedule has at least one job from set $\left\{J_{1}, J_{2}, J_{3}\right\}$, then the following inequality holds:

$$
C_{\max }^{O P T}(L) \geq p_{3}+p_{5}+p_{6}+p_{7}
$$

Hence we get

$$
\frac{C_{\max }^{S}(L)}{C_{\max }^{O P T}(L)} \leq \frac{p_{2}+p_{3}+p_{6}+p_{7}}{p_{3}+p_{5}+p_{6}+p_{7}} \leq \frac{p_{2}+3}{p_{5}+3} \leq \frac{r+3}{4} \leq \frac{r+2}{3} .
$$

Otherwise the optimal schedule is that $\left\{J_{1}, J_{2}, J_{3}\right\}$ and $\left\{J_{4}, J_{5}, J_{6}, J_{7}\right\}$ are assigned separately on two machines. Let $a=\frac{p_{2}+p_{3}}{2}, b=\frac{p_{4}+p_{5}}{2}$, $c=\frac{p_{6}+p_{7}}{2}$. It is easy to see that $a \geq b \geq c$ holds. We analyze the following two cases. In the case of $3 a \leq 2 b+2 c$, we get

$$
\frac{C_{\max }^{S}(L)}{C_{\max }^{O P T}(L)} \leq \frac{p_{2}+p_{3}+p_{6}+p_{7}}{p_{4}+p_{5}+p_{6}+p_{7}}=\frac{a+c}{b+c} \leq \frac{2 b+5 c}{3 b+3 c}=\frac{2}{3}+\frac{c}{b+c} \leq \frac{7}{6},
$$

the last inequality results from $b \geq c$.

In the case of $3 a>2 b+2 c$ we get

$$
\frac{C_{\max }^{S}(L)}{C_{\max }^{O P T}(L)} \leq \frac{p_{2}+p_{3}+p_{6}+p_{7}}{p_{1}+p_{2}+p_{3}}=\frac{2 a+2 c}{3 a}=\frac{2}{3}+\frac{2 c}{3 a} \leq \frac{2}{3}+\frac{2 c}{2 b+2 c} \leq \frac{7}{6} .
$$

For $n=8$, there are exactly four jobs assigned on each machine and there is a machine on which at least two jobs from $\left\{J_{1}, J_{2}, J_{3}\right\}$ are assigned in any optimal schedule. Hence the following inequality holds:

$$
C_{\max }^{O P T}(L) \geq \max \left\{p_{2}+p_{3}+p_{7}+p_{8}, p_{1}+p_{6}+p_{7}+p_{8}\right\} .
$$

Therefore if $L_{1} \geq L_{2}$ we get

$$
\frac{C_{\max }^{S}(L)}{C_{\max }^{O P T}(L)} \leq \frac{p_{1}+p_{4}+p_{5}+p_{8}}{p_{2}+p_{3}+p_{7}+p_{8}} \leq \frac{p_{1}+p_{4}+p_{5}+p_{8}}{p_{7}+p_{4}+p_{5}+p_{8}} \leq \frac{r+3}{4} \leq \frac{r+2}{3} .
$$

If $L_{1} \leq L_{2}$ we get

$$
\frac{C_{\max }^{S}(L)}{C_{\max }^{O P T}(L)} \leq \frac{p_{2}+p_{3}+p_{6}+p_{7}}{p_{1}+p_{6}+p_{7}+p_{8}} \leq \frac{p_{2}+p_{3}+p_{6}+p_{7}}{p_{8}+p_{3}+p_{6}+p_{7}} \leq \frac{r+3}{4} \leq \frac{r+2}{3} .
$$


By the conclusions above, we get

$$
\frac{C_{\max }^{S}(L)}{C_{\max }^{O P T}(L)} \leq \max \left\{\frac{7}{6}, \frac{r+2}{3}\right\}= \begin{cases}\frac{2+r}{3}, & \frac{3}{2}<r \leq 2 \\ \frac{7}{6}, & \frac{4}{3} \leq r \leq \frac{3}{2}\end{cases}
$$

Hence (1) is true for $\frac{4}{3} \leq r \leq 2$.

Now we consider the case of $1 \leq r \leq \frac{4}{3}$ according to the four cases of $n=4 k+1,4 k+2,4 k+3,4 k+4, k=0,1,2, \cdots$. In the following, we will use $S_{i}$ and $S_{i}^{*}$ to denote the job set assigned on machine $M_{i}$ by algorithm $S$ and optimal algorithm, respectively.

For the case of $n=4 k+1$, we have $\left|S_{1}\right|=2 k+1,\left|S_{2}\right|=2 k$, $\max \left\{\left|S_{1}^{*}\right|,\left|S_{2}^{*}\right|\right\} \geq 2 k+1$. Without loss of generality, suppose $\left|S_{1}^{*}\right| \geq 2 k+1$. Then it is easy to see that there exists $i \in\{1,2\}$ satisfying $\left|S_{1}^{*} \cap S_{i}\right| \geq k+1$. If

$$
\begin{aligned}
& \left|S_{1}^{*} \cap S_{1}\right| \geq k+1 \text {, then }\left|S_{1} \backslash S_{1}^{*}\right| \leq k \text {. By } \\
& \quad L_{2}-L_{1}=p_{2}+p_{3}-p_{1}+\sum_{j=1}^{k-1}\left(p_{4 j+2}+p_{4 j+3}-p_{4 j}-p_{4 j+1}\right)-p_{4 k}-p_{4 k+1} \leq 0,
\end{aligned}
$$

we get $L_{1} \geq L_{2}$. Therefore

$$
\begin{aligned}
\frac{C_{\max }^{S}(L)}{C_{\max }^{O P T}(L)} & =\frac{L_{1}}{C_{\max }^{O P T}(L)} \leq \frac{\sum_{j \in S_{1}} p_{j}}{\sum_{j \in S_{1}^{*}} p_{j}} \leq \frac{\sum_{j \in S_{1} \cap S_{1}^{*}} p_{j}+\sum_{j \in S_{1} \backslash S_{1}^{*}} p_{j}}{\sum_{j \in S_{1} \cap S_{1}^{*}} p_{j}+\sum_{j \in S_{1}^{*} \backslash S_{1}} p_{j}} \\
& \leq \frac{\left|S_{1} \cap S_{1}^{*}\right|+\left|S_{1} \backslash S_{1}^{*}\right| r}{\left|S_{1} \cap S_{1}^{*}\right|+\left|S_{1}^{*} \backslash S_{1}\right|} \\
& \leq \frac{\left|S_{1} \cap S_{1}^{*}\right|+\left(\left|S_{1}\right|-\left|S_{1} \cap S_{1}^{*}\right|\right) r}{2 k+1} \\
& \leq \frac{k r+k+1}{2 k+1} \leq \frac{r+1}{2} .
\end{aligned}
$$

If $\left|S_{1}^{*} \cap S_{1}\right| \leq k$, then $\left|S_{1}^{*} \cap S_{2}\right| \geq k+1$. By

$$
L_{1}-L_{2}=p_{1}+\sum_{j=1}^{k}\left(p_{4 j}+p_{4 j+1}-p_{4 j-2}-p_{4 j-3}\right) \leq p_{1}
$$

we get $L_{1} \leq L_{2}+p_{1}$. Therefore

$$
\begin{aligned}
\frac{C_{\max }^{S}(L)}{C_{\max }^{O P T}(L)} & \leq \frac{L_{2}+p_{1}}{C_{\max }^{O P T}(L)} \leq \frac{\sum_{j \in S_{2}} p_{j}}{\sum_{j \in S_{1}^{*}} p_{j}} \leq \frac{\sum_{j \in S_{2} \cap S_{1}^{*}} p_{j}+\sum_{j \in S_{2} \backslash S_{1}^{*}} p_{j}+p_{1}}{\sum_{j \in S_{2} \cap S_{1}^{*}} p_{j}+\sum_{j \in S_{1}^{*} \backslash S_{2}} p_{j}} \\
& \leq \frac{\left|S_{2} \cap S_{1}^{*}\right|+\left|S_{2} \backslash S_{1}^{*}\right| r+r}{\left|S_{2} \cap S_{1}^{*}\right|+\left|S_{1}^{*} \backslash S_{2}\right|} \\
& \leq \frac{\left|S_{2} \cap S_{1}^{*}\right|+\left(\left|S_{2}\right|-\left|S_{2} \cap S_{1}^{*}\right|\right) r+r}{2 k+1} \\
& \leq \frac{k r+k+1}{2 k+1} \leq \frac{r+1}{2} .
\end{aligned}
$$


For the case of $n=4 k+2$, we have $\left|S_{1}\right|=2 k+1,\left|S_{2}\right|=2 k+1$, $\max \left\{\left|S_{1}^{*}\right|,\left|S_{2}^{*}\right|\right\} \geq 2 k+1$. Without loss of generality, suppose $\left|S_{1}^{*}\right| \geq 2 k+1$. Therefore there exists $i \in\{1,2\}$ satisfying $\left|S_{1}^{*} \cap S_{i}\right| \geq k+1$. In the following we consider this case according to the two subcases of $L_{1} \geq L_{2}$ and $L_{1}<L_{2}$.

In this case of $L_{1} \geq L_{2}$, if $\left|S_{1}^{*} \cap S_{1}\right| \geq k+1$ holds, then the following is true:

$$
\begin{aligned}
\frac{C_{\max }^{S}(L)}{C_{\max }^{O P T}(L)} & =\frac{L_{1}}{C_{\max }^{O P T}(L)} \leq \frac{\sum_{j \in S_{1}} p_{j}}{\sum_{j \in S_{1}^{*}} p_{j}} \leq \frac{\sum_{j \in S_{1} \cap S_{1}^{*}} p_{j}+\sum_{j \in S_{1} \backslash \backslash_{1}^{*}} p_{j}}{\sum_{j \in S_{1} \cap S_{1}^{*}} p_{j}+\sum_{j \in S_{1}^{*} \backslash S_{1}} p_{j}} \\
& \leq \frac{\left|S_{1} \cap S_{1}^{*}\right|+\left|S_{1} \backslash S_{1}^{*}\right| r}{\left|S_{1} \cap S_{1}^{*}\right|+\left|S_{1}^{*} \backslash S_{1}\right|} \\
& \leq \frac{\left|S_{1} \cap S_{1}^{*}\right|+\left(\left|S_{1}\right|-\left|S_{1} \cap S_{1}^{*}\right|\right) r}{2 k+1} \\
& \leq \frac{k r+k+1}{2 k+1} \leq \frac{r+1}{2} .
\end{aligned}
$$

If $\left|S_{1}^{*} \cap S_{1}\right| \leq k$ holds, we consider the following two subcases of $\left|S_{1}^{*}\right|=2 k+1$ and $\left|S_{1}^{*}\right| \geq 2 k+2$.

For the case of $\left|S_{1}^{*}\right|=2 k+1,\left|S_{2}^{*}\right|=2 k+1$ holds by $n=4 k+2$. By $\left|S_{1}\right|=2 k+1$ and $\left|S_{1}^{*} \cap S_{1}\right| \leq k$ we get $\left|S_{2}^{*} \cap S_{1}\right| \geq k+1$. Therefore

$$
\begin{aligned}
\frac{C_{\max }^{S}(L)}{C_{\max }^{O P T}(L)} & =\frac{L_{1}}{C_{\max }^{O P T}(L)} \leq \frac{\sum_{j \in S_{1}} p_{j}}{\sum_{j \in S_{2}^{*}} p_{j}} \leq \frac{\sum_{j \in S_{1} \cap S_{2}^{*}} p_{j}+\sum_{j \in S_{1} \backslash S_{2}^{*}} p_{j}}{\sum_{j \in S_{1} \cap S_{2}^{*}} p_{j}+\sum_{j \in S_{2}^{*} \backslash S_{1}} p_{j}} \\
& \leq \frac{\left|S_{1} \cap S_{2}^{*}\right|+\left|S_{1} \backslash S_{2}^{*}\right| r}{\left|S_{1} \cap S_{2}^{*}\right|+\left|S_{2}^{*} \backslash S_{1}\right|} \\
& \leq \frac{\left|S_{1} \cap S_{2}^{*}\right|+\left(\left|S_{1}\right|-\left|S_{1} \cap S_{2}^{*}\right|\right) r}{2 k+1} \\
& \leq \frac{k r+k+1}{2 k+1} \leq \frac{r+1}{2} .
\end{aligned}
$$

For the case of $\left|S_{1}^{*}\right| \geq 2 k+2$, by $\left|S_{1}^{*} \cap S_{1}\right| \leq k$ we get $\left|S_{1}^{*} \cap S_{2}\right| \geq k+2$. By rules of $S$ algorithm, we have

$$
L_{1}-L_{2}=p_{1}+\sum_{j=1}^{k}\left(p_{4 j}+p_{4 j+1}-p_{4 j-2}-p_{4 j-1}\right)-p_{4 k+2} \leq p_{1}-p_{4 k+2} .
$$

That means $L_{1} \leq L_{2}+p_{1}-p_{4 k+2}$. Therefore

$$
\begin{aligned}
& \frac{C_{\max }^{S}(L)}{C_{\max }^{O P T}(L)}=\frac{L_{1}}{C_{\max }^{O P T}(L)} \leq \frac{L_{2}+p_{1}-p_{4 k+2}}{C_{\max }^{O P T}(L)} \\
& \leq \frac{\sum_{j \in S_{2} \cap S_{1}^{*}} p_{j}+\sum_{j \in S_{2} \backslash S_{1}^{*}} p_{j}+p_{1}-p_{4 k+2}}{\sum_{j \in S_{2} \cap S_{1}^{*}} p_{j}+\sum_{j \in S_{1}^{*} \backslash S_{2}} p_{j}} \leq \frac{\left|S_{2} \cap S_{1}^{*}\right|+\left|S_{2} \backslash S_{1}^{*}\right| r+r-1}{\left|S_{2} \cap S_{1}^{*}\right|+\left|S_{1}^{*} \backslash S_{2}\right|} \\
& \leq \frac{\left|S_{2} \cap S_{1}^{*}\right|+\left(\left|S_{2}\right|-\left|S_{2} \cap S_{1}^{*}\right|\right) r+r-1}{2 k+2} \leq \frac{k r+k+1}{2 k+2} \leq \frac{k r+k+1}{2 k+1} \leq \frac{r+1}{2} .
\end{aligned}
$$


Similarly we can prove the case of $L_{1}<L_{2}$.

By the same way used above, we can also show that (1) is true for the case of $n=4 k+3$ and $n=4 k+4$ for $1 \leq r \leq \frac{4}{3}$. Now we show the tightness of the bound.

$$
\text { For } \frac{3}{2}<r \leq 2 \text {, Let } L^{(1)}=\left\{J_{1}, J_{2}, J_{3}, J_{4}, J_{5}\right\} \text { with } p_{1}=r, p_{i}=1, i=2, \cdots, 5 \text {. By }
$$

the rules of $S$ algorithm, we have $L_{1}=r+2, L_{2}=2$, i.e., $C_{\max }^{S}\left(L^{(1)}\right)=r+2$. It is easy to see that $C_{\max }^{O P T}\left(L^{(1)}\right)=3$. Hence

$$
\frac{C_{\max }^{S}\left(L^{(1)}\right)}{C_{\max }^{O P T}\left(L^{(1)}\right)}=\frac{r+2}{3} .
$$

It is easy to show the tightness for $\frac{4}{3} \leq r \leq \frac{3}{2}$ by job list

$L^{(2)}=\left\{J_{1}, J_{2}, J_{3}, J_{4}, J_{5}, J_{6}, J_{7}\right\} \quad$ with $\quad p_{1}=p_{2}=p_{3}=\frac{4}{3}, \quad p_{4}=p_{5}=p_{6}=p_{7}=1$

and for $1 \leq r \leq \frac{4}{3}$ by job list with $p_{1}=p_{2}=p_{3}=r, p_{4}=p_{5}=p_{6}=p_{7}=1$.

\section{Concluding Remarks}

In this paper, we consider ordinal on-line scheduling for jobs with known sizes in $[1, r](r \geq 1)$ and non-decreasing processing times on two parallel machines system. Firstly it is proved that the worst case performance ratio of the existing algorithm $P_{2}$ can not be improved even if the job processing times are known in $[1, r]$ for any $r \geq 1$. Secondly, an algorithm named $S$ is proposed and its worst case performance ratio is given as follow:

$$
R(2, S)= \begin{cases}\frac{2+r}{3}, & \frac{3}{2}<r \leq 2 \\ \frac{7}{6}, & \frac{4}{3} \leq r \leq \frac{3}{2} \\ \frac{r+1}{2}, & 1 \leq r<\frac{4}{3}\end{cases}
$$

which is better than algorithm $P_{2}$. Just two machines are considered here. It is an interesting problem to consider general $m$ machines system to design better algorithm.

\section{Acknowledgements}

The authors would like to express their thanks to the National Natural Science Foundation of China for financially supporting under Grant No. 11471110 and the Foundation Grant of Education Department of Hunan (No. 16A126).

\section{Conflicts of Interest}

The authors declare no conflicts of interest regarding the publication of this paper. 


\section{References}

[1] Graham R.L. (1969) Bounds on Multiprocessing Timing Anomalies. SIAM Journal on Applied Mathematics, 17, 416-429. https://doi.org/10.1137/0117039

[2] Cheng, T.C.E., Kellerer, H. and Kotov, V. (2012) Algorithms Better than LPT for Semi-Online Scheduling with Decreasing Processing Times. Operations Research Letters, 40, 349-352.

[3] He, Y. and Dósa, G. (2005) Semi-Online Scheduling Jobs with Tightly-Grouped Processing Times on Three Identical Machines. Discrete Applied Mathematics, 150, 140-159.

[4] He, Y. and Zhang, G. (1999) Semi on-Line Scheduling on Two Identical Machines. Computing, 62, 179-187. https://doi.org/10.1007/s006070050020

[5] Kellerer, H., Kotov, V., Speranza, M.G. and Tuza, Z. (1997) Semi on-Line Algorithms for the Partition Problem. Operations Research Letters, 21, 235-242. https://doi.org/10.1016/S0167-6377(98)00005-4

[6] Lin, L. and Tan, Z. (2014) Inefficiency of Nash Equilibrium for Scheduling Games with Constrained Jobs: A Parametric Analysis. Theoretical Computer Science, 521, 123-134. https://doi.org/10.1016/j.tcs.2013.11.012

[7] Gupta, S., Dalal, U.D. and Mishra, V.N. (2014) Novel Analytical Approach of Conventional Mapping Scheme with Discrete Hartley Transform in OFDM System. American Journal of Operations Research, 4, 281-292. https://doi.org/10.4236/ajor.2014.45027

[8] Liu, W.P., Sidney, J.B. and Vliet, A.V. (1996) Ordinal Algorithms for Parallel Machine Scheduling. Operations Research Letters, 18, 223-232.

https://doi.org/10.1016/0167-6377(95)00058-5 